\title{
Audit of height measurement at age 3 years: results of a survey of Scottish health boards
}

\author{
A D Allen, A $M$ Wallace
}

\begin{abstract}
Height measurement at about the age of 3 years is accepted as a routine practice by all 15 Scottish health boards and is a recommendation of the Hall report Health for all Children. As part of a Scotlandwide project to assess the feasibility of audit of preschool surveillance programmes using routinely collected data, all boards were asked for information about this procedure. The results show that, while all boards confirmed its usefulness as a screening measure, only one board was realistically able to audit height measurement at this age at all stages using routinely available data.
\end{abstract}

The whole screening process, including programme management, equipment validation, staff training and referral criteria, was examined using the quality standards defined in the Hall report. Results showed a wide variation between boards. For example, fewer than half of the boards provided guidelines for height measurement at age 3 to all professionals involved.

The availability of even basic outcome data, such as numbers of children measured at this age was patchy, although this will improve with the introduction of the national computerised preschool surveillance system. Two boards have no plans to record such data routinely. In conclusion, before outcome data can be used and relied on, health boards and trusts need to develop local guidelines including quality standards such as age limits for measurement, programme management, provision of equipment, and review and referral criteria for inclusion into contracts.

(Arch Dis Child 1996; 74: 332-335)

Keywords: height measurement, preschool surveillance, clinical audit, quality standards.

A one year project was undertaken in 1993, funded by the Clinical Resource and Audit Group of the Scottish Office, to examine the feasibility of auditing preschool child health surveillance programmes in Scottish health boards, whether locally or on a Scotland-wide basis. As part of this it was decided to look in detail at two specific screening tests, chosen as sensitive conditions to reflect the overall effectiveness of the programmes. One of these was height measurement at age 3 years.

The survey was prompted by significant changes affecting preschool child health surveillance in Scotland since 1990:

(1) The introduction of the internal market into the NHS structure, and in particular general practitioner (GP) contracts for child health surveillance, meant that by 1993 the majority of preschool child health surveillance was being undertaken in GP rather than child health clinics.

(2) There had been the important re-evaluation of child health surveillance in the report of the Joint Working Party on Child Health Surveillance Health for all Children, ${ }^{1}$ the recommendations of which were commended to Scottish health boards by the Scottish Office in March 1992.

(3) There had also been substantial progress towards improvements in data collection for child health surveillance through computerised systems. While two health boards had developed their own computer systems for collection of child health surveillance data, for the majority of health boards the national computerised child health surveillance programme was being developed.

The aim of this system, based on the community health index, was to record all aspects of the surveillance programme, administrative arrangements, clinical findings, and reports on results and outcomes. It is also potentially linked with other records systems, for example the special needs system.

Clinical audit requires comprehensive, comparable, and reliable data, based on a clearly defined standard for the procedure being provided. The survey of height measurement at age 3 therefore aimed to look at quality using the standards defined in the Hall report as accepted good practice (see table 1 ).

Height (and length) measurement is a routine procedure at most check ages in all Scottish health boards as part of preschool child health surveillance. Its value lies in two main areas: early detection of treatable conditions and monitoring the general health of the population. It has also a potential role as an indicator of the effectiveness of surveillance programmes. However, its role as a screening procedure has been questioned, ${ }^{2}$ and there are a number of views on the frequency and optimum age for height measurement, both in terms of the results achieved and the cost.

The participants in the Tayside growth study have commented: 'We believe that monitoring growth in children is one of the most sensitive measures of physical, emotional and social well-being'. ${ }^{3}$

\section{Methods}

All Scottish health boards were sent a questionnaire. The responses were subsequently recorded by the audit assistant at meetings 
Table 1 Height measurement at age 3: quality standards based on the recommendations of the Hall report ${ }^{1}$

\begin{tabular}{|c|c|}
\hline Management & $\begin{array}{l}\text { One person to be nominated to have formal overall responsibility } \\
\text { for the programme. }\end{array}$ \\
\hline $\begin{array}{l}\text { Test protocol } \\
\text { Age of measurement }\end{array}$ & $\begin{array}{l}\text { Test protocol to be available to all staff. } \\
\text { (1) 'The optimum age for measurement of height as a universal } \\
\text { screening procedure is around the third birthday'. } \\
\text { (2) Stated age limits for performance of the test, to facilitate } \\
\text { comparability of data. }\end{array}$ \\
\hline $\begin{array}{l}\text { Recording of results } \\
\text { Provision of equipment }\end{array}$ & $\begin{array}{l}\text { Height of every child measured to be plotted on a centile chart. } \\
\text { (1) For best results, device such as a Minimetre or Magnimetre } \\
\text { to be used. } \\
\text { (2) Equipment to be correctly installed and maintained. }\end{array}$ \\
\hline Staff training & $\begin{array}{l}\text { Training and updating required in all aspects of protocol. } \\
\text { Should be defined. }\end{array}$ \\
\hline $\begin{array}{l}\text { Pattern of review and } \\
\text { referral }\end{array}$ & Should be a consistent pattern defined for all practitioners. \\
\hline $\begin{array}{l}\text { Action where child } \\
\text { appears to be short }\end{array}$ & $\begin{array}{l}\text { Should be explicit policy on further measurement, and on } \\
\text { responsibility for referrals. }\end{array}$ \\
\hline
\end{tabular}

with representatives of the health boards. Copies of the completed responses were returned to boards for final approval.

The questionnaire was devised with the assistance of a small group consisting of a consultant in public health medicine, a GP, a senior clinical medical officer, and a deputy chief nursing officer, all from different health boards.

The qualitative information sought from health boards was based on the recommendations of the Hall report (see table 1).

All 15 health boards were also asked about availability of quantitive information on results and outcomes of height measurement at age 3 years, as both numbers and percentages of the target population, as appropriate. The items covered were as follows:

- Children screened

- Children screened on or below the 3rd centile* for height

- Children referred as a consequence of screening

- After referral, children found to be on or below the 3rd centile` for height

- Children found not to require further specialist follow up

- Children with a condition affecting their growth and requiring treatment

- Route these children were discovered

- Average age of diagnosis of main conditions affecting growth

- Distribution of height measurements of local population at this age in comparison with national standards.

\section{Results}

Results showed:

- That all Scottish health boards supported the provision of height measurement for every child between the ages of 36 and 42 months.

- That the vast majority of height measurements are performed by health visitors, $100 \%$ in eight boards, $80-90 \%$ in five boards.

- One board (Dumfries and Galloway) had audited height measurement of preschool children with the aim of establishing the incidence of short stature in their area, and to ascertain the outcome of the children involved. In addition, the Tayside growth study had

*Note: The study was undertaken before the publication in October 1993 of upwardly revised national growth standards. ${ }^{4}$ conducted an audit of management of short stature covering all age ranges: height data obtained had been incorporated into new national height standards. ${ }^{3}$

\section{QUALITY STANDARDS}

The quality standards in each of the health boards compared with those defined in the Hall report were as follows:

\section{Management}

No board had adopted the recommendation of the Hall report that one person should be nominated to manage the provision of preschool child health surveillance as a whole, together with individuals for specific aspects of the programme.

\section{Test protocol}

One board had a test protocol covering all the elements of quality standards, and available to all relevant staff. Eight boards had no such protocol. Six boards had protocols, formal or informal, covering some of the information or available to some of the staff.

\section{Age of measurement}

All boards accepted the recommendation of the Hall report that around the age of 3 was the optimum age for measurement of height as a universal screening procedure. Seven of 15 boards had stated time limits for the performance of the 39-42 month check (including height measurement).

\section{Training and test conditions}

Table 2 summarises the availability of these conditions for height measurement at age 3 . The results show that the attention paid to all these aspects of this screening procedure is very patchy.

\section{Stated criteria for a positive test result}

All boards agreed that a positive screening result would be a height measurement on or below the 3rd centile, or a significant downward trend through the centiles. In at least seven boards however this was not formally stated.

\section{Exclusion criteria}

Should be defined so that each practitioner is as far as possible screening the same group of children. Most boards excluded children unable to be measured for physical reasons and some suggested exclusion of children already under specialist care. In most boards however there was no formal statement of which children should be not be measured.

\section{Review and referral}

Two boards provided guidelines on review and referral of short children, along the lines of 
Table 2 Height measurement at age 3: availability of quality standards and training and test conditions from 15 Scottish health boards

\begin{tabular}{lc}
\hline & $\begin{array}{c}\text { Elements } \\
\text { available }\end{array}$ \\
\hline Equipment: availability of Minimetre & \\
In all or most clinics & 3 \\
In all or most child health clinics: GP clinics not known & 4 \\
No policy/do not know what is used & 8 \\
Accuracy of equipment & 0 \\
Routine checks on accuracy of equipment & 8 \\
Recording of results: centile charts & 4 \\
Every child's height plotted on A4 size Tanner-Whitehouse growth chart & 1 \\
Every child's height plotted in PHR/Woodside record: full size chart if concerned & 1 \\
Height plotted on chart only if concerned & 1 \\
No specific policy & 2 \\
Meights plotted at child health clinics: GP clinics not known & 5 \\
Precise instructions provided & 8 \\
General instructions available to some staff & \\
Instructions not provided & 0 \\
Reliability of individuals' measurement technique & \\
Individual personnel asked to assess reliability of their measurements & 2 \\
Health visitors' in-service refresher sessions & 9 \\
Regular voluntary in-service refresher session & 4 \\
Occasional study days & \\
No provision/not known & 1 \\
GPs' in-service refresher sessions & 1 \\
As part of annual update on child health surveillance (voluntary) & 1 \\
As part of two day course for trainee GPs (mandatory) & 12 \\
Voluntary occasional courses & 0 \\
Not provided (except in some cases in accreditation courses) & 3 \\
'Designated officer' & 12 \\
Assumed to be health visitors or nurse managers solely & \\
Assumed to be shared: GPs or child health staff and health visitors & \\
\hline
\end{tabular}

*The Leicester Height Measure recently introduced: both this and minimetre recommended by the British Society for Paediatric Endocrinology.

PHR=parent held record.
Whether referral was direct from GP/clinical medical officer to growth specialist, or initially to another paediatrician, depended mainly on geographical factors. All boards had access to the four main centres for growth treatment at Aberdeen, Dundee, Edinburgh, and Glasgow.

\section{QUANTITATIVE DATA}

Table 3 summarises the availability of routinely available quantitative data on height measurement at age 3. It indicates that in the autumn of 1993 only one board could obtain all the items of data that might be regarded as necessary to assess the effectiveness of height measurement as a screening procedure. Looking at basic items of coverage, only six of 15 boards had available data on the number of children whose height was measured at that age, and only five out of 15 could ascertain the number and percentage of children in the target population screened on or below the 3rd centile for height. Additionally, in the three boards piloting the child health surveillance programme system the data was potentially available when the system had been in place for a sufficient length of time. Two boards had no plans to collect this data routinely.

recommendations of the Hall report. These suggested:

- Immediate referral if a child appeared very short or was outside target range.

- Otherwise remeasurement in six months' time and consideration of referral in light of growth velocity obtained.

In the remaining 13 boards practice on review and referral was varied and there was no formal pattern.

- In most boards, the health visitor decided whether to seek the advice of the GP/clinical medical officer or whether to remeasure at a later date.

- The criteria for review or referral were not stated and the remeasurement interval was not specified.

No other checks or tests were routinely undertaken, although parental heights might be taken into account (but not necessarily through calculation of a mid-parental centile).

Table 3 Height measurement at age 3: availability of results and outcomes at October 1993 from 15 Scottish health boards

\begin{tabular}{|c|c|c|c|}
\hline & $\begin{array}{l}\text { Data } \\
\text { available }\end{array}$ & $\begin{array}{l}\text { Data } \\
\text { potentially } \\
\text { available }\end{array}$ & $\begin{array}{l}\text { Data } \\
\text { not } \\
\text { available }\end{array}$ \\
\hline \multicolumn{3}{|l|}{ Coverage (as number and percentage of target population) } & 6 \\
\hline Results (as number and percentage of target population) & & & \\
\hline $\begin{array}{l}\text { Children screened on or below 3rd centile } \\
\text { Outcomes (as number and percentage of target population) }\end{array}$ & 5 & 3 & 7 \\
\hline $\begin{array}{l}\text { Children referred as a consequence of screening } \\
\text { After referral children found to be on or below the 3rd }\end{array}$ & 5 & 3 & 7 \\
\hline centile for height & 5 & 3 & 7 \\
\hline Children found not to require further specialist follow up & 5 & 3 & 7 \\
\hline \multicolumn{4}{|l|}{ Overall outcomes } \\
\hline affecting their growth and requiring specialist treatment & 5 & 3 & 7 \\
\hline $\begin{array}{l}\text { If available can route condition detected be analysed? } \\
\text { Average age of diagnosis of main conditions affecting }\end{array}$ & 5 & & \\
\hline growth? & 2 & 3 & 10 \\
\hline $\begin{array}{l}\text { Comparison of local height distribution with national } \\
\text { standards }\end{array}$ & 1 & 3 & 11 \\
\hline
\end{tabular}

\section{Discussion}

Height measurement around the age of 3 years is a highly valued, commonly used screening technique in Scotland. Nevertheless, little attention is given by most health boards to the formal development of quality standards or to the collection of data to measure effectiveness.

The notion that height measurement is a low technology screening test has led to the assumption of an (acceptable) performance variation in equipment and training in the community setting. One study which looked at measurement by health visitors found this assumption justified ${ }^{5}$; others have shown the need for continuing awareness of problems both in the choice of equipment and the standards of measurement technique in carrying out this apparently simple technique. ${ }^{6}{ }^{7}$ No board had considered the regular calibration of measuring devices. Assuming height measurement devices are correctly placed can lead to significant unreliability in height measurement. $^{6}$

There was a lack of clarity as to who had overall responsibility to effect any necessary changes to conditions and who should make arrangements to buy new equipment when required. In most boards, training is largely limited to occasional training sessions with only two boards organising regular sessions.

There are wide variations in the use of charts both among the majority who provided a chart of some sort for all children and the remaining three boards who either had no clear policy or only provided a chart where there was concern. A consistent policy for the accurate recording of results is an essential part of height measurement as a screening process to establish a baseline and monitor the pattern of growth for every child. 
Few boards define clear criteria for children who should be excluded from the measurement population. Children physically unable to stand may be an obvious exclusion, but there may need to be a clear statement on whether children being followed up (whether for growth or another reason) should or should not be included in screening. There may be a danger of a child not being screened as it is assumed someone else is doing it. One of the aims of collecting height data is to obtain comprehensive information on all children to assess the profile for the area. This will not be achieved if data on short children are omitted.

In a number of boards there is no clear statement of the appropriate cut off point that would be necessary if height measurement is to be treated as a screening procedure. Any such statement may need to be qualified to make clear that further follow up need not be confined only to children whose screening height measurement puts them on or below the relevant cut off point, so that the clinical judgment of the practitioner is not superseded by a measurement which may or may not be correct. In addition, any child whose height measurement has fallen significantly through the centiles should be followed up whatever the height measurement.

The result of the survey showed that in all but two boards practice in review and referral was varied and there was no stated or consistent formal pattern. The onus of review or referral falls in the first place mainly on health visitors and clear guidelines are needed on what to do when the measurement suggests that a child is short.

Any guideline recommending the use of growth velocity in the community needs to be treated with caution. At least three studies have highlighted the extreme caution with which velocity calculations need to be approached at community level. ${ }^{7-9}$ Growth velocity is a useful tool for the specialists, but uncertainties remain about the variations in growth rates; there are also wide confidence intervals to be allowed on velocity calculations, especially at the level of accuracy available from community screening measurements. It may be more appropriate for guidelines on height measurement as a screening procedure to focus on growth trend rather than velocity.

The question of what action to take where child appears to be short has formed part of the major research project on Tayside, ${ }^{3}$ which has conducted an audit of guidelines for the management of the short child. In this study a special growth chart for all children identified as being on or below the 3rd centile, with guidelines as to management, was supplied to the primary care practitioner involved. The results did not indicate that GPs found the guidelines widely useful.

An alternative approach has been tried in Oxford, ${ }^{9}$ which has offered all preschool children on or below the 3rd centile and not already being followed up elsewhere a second level of growth monitoring and assessment by an auxologist. This has resource implications.

The stated aim of the survey was to assess the feasibility of audit. It was not possible to undertake an audit of the effectiveness of height measurement using routinely collected data as it became obvious during the preparatory stages of the survey that the majority of boards would not be able to provide most of the necessary data. This was also demonstrated by the survey.

Much attention is being given to collecting data in the field of preschool child health surveillance with the development of the new national computerised child health surveillance programme. It is being adopted by the majority of health boards at the present time. Nevertheless, until the quality issues are addressed in a systematic and formal way the value of the data may be called into question.

The quality standards addressed in this paper including test conditions, training and criteria for exclusion, review and referral have been clearly defined by Hall and are widely accepted and, indeed were commended to boards by the Scottish Office in 1992. It is now for health boards and trusts to develop local guidelines to be incorporated within the contracts to ensure this relatively simple screening procedure can be used to its full potential.

We ackowledge with thanks the support of the Clinical Resource and Audit Group of the Scottish Office in funding this project.

1 Hall DMB, ed. Joint Working Party on Child Health Surveillance. Health for all children. 2nd Ed. Oxford Oxford University Press, 1991. (Hall report.)

2 Macfarlane JA. Should we screen for growth problems in children? The pragmatic answer for 1994-5. Fournal of Medical Screening 1994; 1: 50-4.

3 White E. Growth of children in Tayside. Report of Tayside growth study. Dundee: Department of Child Health, Ninewells Hospital and Medical School, 1994

4 Cole TJ. Do growth chart centiles need a facelift? $B M F$ 1994; 308: $641-2$.

5 Ahmed ML, Yudkin PL, Macfarlane JA, McPherson K Dunger DB. Are measurements of height made by health visitors sufficiently accurate for routine screening of visitors sufficiently accurate for routine
growth? Arch Dis Child 1990; 65: 1345-8.

6 Voss LD, Bailey BJR, Cumming K, Wilkin TJ, Betts PR.The reliability of height measurement (the Wessex growth reliability of height measurement (the
study). Arch Dis Child 1990; 65: 1340-4.

7 Cotterill AM, Majrowski WH, Hearn SJ, Jenkins S, Savage MO. Assessment of the reliability of school nurse heigh measurements in an inner-city population (the Hackney growth initiative). Child Care Health Dev 1993; 19: 159-65.

8 Voss LD, Wilkin TJ, Bailey BJR, Betts PR. The reliability of height and height velocity in the assessment of growth (the Wessex growth study). Arch Dis Child 1991; 66: 833-7.

9 Ahmed ML, Allen AD, Sharma A, Macfarlane JA, Dunger DB. Evaluation of a district growth screening programme: the Oxford growth study. Arch Dis Child 1993; 69: 361-5. 